\title{
The permissive non-parental childcare and moral intelligence: Indonesian setting
}

\author{
Falakhul Auliya', Yuli Kurniawati Sugiyo Pranoto², Sunawan ${ }^{3}$, Ali Sunarso ${ }^{4}$ \\ ${ }^{1}$ Department of Early Childhood Education, Postgraduate Program, Universitas Negeri Semarang, Indonesia \\ ${ }^{2}$ Department of Teacher Education for Early Childhood Education, Universitas Negeri Semarang, Indonesia \\ ${ }^{3}$ Department of Guidance and Counseling, Universitas Negeri Semarang, Indonesia \\ ${ }^{4}$ Department of Primary Teacher Education, Universitas Negeri Semarang, Indonesia
}

\begin{tabular}{l}
\hline Article Info \\
\hline Article history: \\
Received Sep 12, 2020 \\
Revised Mar 22, 2021 \\
Accepted Apr 19, 2021 \\
\hline Keywords: \\
Household assistants \\
Moral intelligence \\
Parenting \\
Permissive parenthood
\end{tabular}

\begin{abstract}
Most parents in Indonesia are unable to provide maximum care for their children while working. Childcare includes fostering moral intelligence, which is carried out by grandmothers, household assistants, and daycare assistants. Furthermore, 178 young children (95 males and 83 females) and 178 caregivers (68 grandmothers, 54 household assistants, and 56 daycare assistants) were involved in the study. Simple regression analysis was used to examine parenthood on moral intelligence, and moderator analysis was used to test permissive non-parental childcare and moral intelligence in early childhood. The results showed that authoritative and permissive parenthood has an effect on moral intelligence and that the effects of permissiveness on moral intelligence are only moderately influenced. Permissive non-parental childcare and moral intelligence apply to the group of grandmothers and household assistants. The research provides a detailed description of the role of predictors on moral intelligence in non-parental care.
\end{abstract}

This is an open access article under the CC BY-SA license.

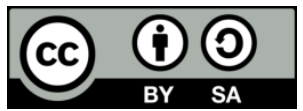

\section{Corresponding Author:}

Falakhul Auliya

Department of Early Childhood Education, Postgraduate Program

Universitas Negeri Semarang

Jl. Kelud Utara III, Semarang 50237, Indonesia

Email: fhalliya@gmail.com

\section{INTRODUCTION}

Moral intelligence is important for human development and in people's lives [1]. At present, it is a very interesting topic because it affects various aspects of human life [2], and it is one of the most important intelligence that should be considered. Research conducted by Janssens and Maja [3] shows that the association between parenting styles and moral behavior of children is constant from childhood to adulthood. Other research examining the moral intelligence of high school students in Nigeria shows that moral intelligence is related to academic integrity [4].

Moral intelligence is the center of human intelligence because it has a great influence on other fields and serves as a common thread for future action [5], [6]. Furthermore, it is an act that represents universal principles and the truths of human behavior [7]. The development of moral intelligence is not only limited to reasoning (moral knowing) but also has to consider other aspects, such as feelings and action [8]-[10]. Moral intelligence refers to the knowledge of the universal goodness and values in the society, while moral feelings relate to loving attitudes and adhering to the values of kindness, which create an awareness for moral behavior [11]. 
Several studies have discussed the impact of parenthood on children's moral intelligence. In the first five years, children need love, especially from both parents. However, compassion is the first thing children learn and teach to show empathy for others. Along with these patterns, moral intelligence becomes very important and a source of compassion. Moral intelligence provide purpose for humans, and people behave in the right way when they have high moral intelligence [12].

Parents play an important role in raising children, especially in fostering moral intelligence. Children's early experiences involving brain development through their interactions with parents and caregivers have a direct influence on moral intelligence [13]. Therefore, parents and caregivers need to provide moral assistance from time to time, considering that it cannot be formed in a very short time.

Research by Campaert, Annalaura, and Ersilia [14] shows that poor parenting has an impact on children's moral detachment, which is usually characterized by aggressive behavior. In addition, the results of research conducted by Augustine and Stifter [15] shows that positive and warm parenting is effective for development of children's conscience and compliance. Gentle parents have the advantage of constantly developing the moral behavior of their children. Meanwhile, research conducted by Karmakar [16] shows that parenting styles that enforce prohibitions and restrictions make children aware of the rules and norms that apply in the family and society. However, this parenting style does not apply to adolescents because parents that apply too many youth restrictions may influence teenagers' behavior in order to adhere to values and rules, as negative consequences are avoided. Research conducted by Alhadabi, et al. [17], shows that a father's upbringing is directly related to moral intelligence, but a mother's upbringing is not directly related to moral intelligence.

Unfortunately, not all children receive more attention from both parents because of responsibility. This is also shown in the work participation rate of women over 15 years of age in Indonesia, which shows an increase from the previous year to 53.08\% [18]. Parents that can not look after or care for their children while working will transfer the responsibility to others (non-parental childcare). Parents in Indonesia that are busy working usually transfer their childcare to other people, such as close relatives (grandparents, uncles, and aunts), household assistants, or daycare [19], [20].

Several previous studies also investigated non-parental care, similar to the research by $\mathrm{Ma}$, Chen, and Li [21] non-parental home care is an important type of childcare that is widely used in the developed societies, which has a substantial effect on the children's development. However, this study focus more on the involvement of foreign domestic servants in non-parental care in Hong Kong. Another study by Zhang, et al. [22] focused on parents in urban Chinese with a 14-month-old child that discussed about caregivers and ideals in caring for the children. The results showed that parents in urban Tionghoa employ caregivers to complement the childcare provided by both grandparents and mothers (multicaregivers) to improve the quality of childcare. De Moura, et al. [23] analyzed potential differences between caregivers, such as mothers, grandmothers, household assistants, and daycare assistants. The results showed that there were significant differences between caregivers, which includes basic care, body stimulation, and personal attention. However, previous research focused more on the differences in non-parental upbringing, than on their impact on some aspects of development, such as aspects of moral development.

Research on non-parental care has not been widely carried out, but the facts regarding the condition of children involved in non-parental care have become a phenomenon. In light of this phenomenon, this study aims to explore non-parental care for the development of moral intelligence, considering that many parents have the potential to entrust childcare to non-parental care. Research on the role of non-parental caregivers in the development of moral intelligence is very important because parents are the strongest moral agents [24], [25]. Previous studies partially address non-parental care and no one has extensively discussed the effects of non-parental care on moral intelligence. Therefore, the research on the effect of non-parental care simultaneously on early childhood moral intelligence is important. This study will contribute to the development of early childhood moral intelligence when non-parental care is involved in childcare routines.

\section{RESEARCH METHOD}

\subsection{Participants}

This study included 178 young children (95 males and 83 females) aged 5-6 years in Semarang City, Central Java, Indonesia. (Mage=5.60, $\mathrm{SD}=0.491$ ) and their caregivers (68 grandmothers, 54 household assistants, and 56 daycare assistants). Children participated voluntarily having the parents' permission and were from 11 kindergartens. The caregivers include grandmothers, household assistants, and daycare assistants that were female. 


\subsection{Measurements}

\subsubsection{Parenting}

Measurement of parenting patterns applied by child caregivers using the Parenting Scale and Dimensions Questionnaire (PSDQ) [26]. The PSDQ scale consists of 62 items with five points $(1=$ never to $5=$ always). PSDQ scale translation uses the back-translation procedure, and the Cronbach's alpha in this study was .75 .

\subsubsection{Moral intelligence}

The scale used for measuring children's moral intelligence was developed based on the theory of Borba's [8], which consists of seven moral virtues. Borba's [8] moral virtues are empathy, conscience, selfcontrol, respect, kindness, tolerance, and justice. This scale consists of 14 illustrations with three score categories, namely (low to high). Each illustration contains three question items (think, feel, do), and the Cronbach's alpha in this study was .75.

\subsection{Procedures}

The research activity started with obtaining parental consent as children and caregivers are to participate. After obtaining the consent, the reporters then conducted a storytelling test to measure the children's moral intelligence using a scale. Furthermore, the storytelling test technique is carried out by face-to-face interviews and orally, considering that early children cannot read fluently. The storytelling test is carried out flexibly according to the school schedule, readiness, and condition of the child. In general, the duration of the test is \pm 10 -30 minutes, but some children need more than 30 minutes. The reporter conducted a storytelling test with a 3-person research team. After collating the children's answers, the children were graded according to their answers, and the caregivers were asked to fill in the parenting scale.

\section{RESULTS}

\subsection{Descriptive data}

Table 1 presents the mean and standard deviation of children's moral intelligence and parenting patterns based on the care group and presents the results of the ANOVA calculation. Furthermore, it also showed that the children's moral intelligence and parenting style in the group of grandmothers, household assistants, and daycare caregivers are in the medium category. The ANOVA calculation results showed that there are significant differences in authoritative childcare in the group of grandmothers and daycare assistants.

Table 1. Mean, standard deviation, and ANOVA

\begin{tabular}{cccccccccc}
\hline & \multicolumn{2}{c}{ Grandmothers $(\mathrm{n}=68)$} & \multicolumn{2}{c}{ Household assistants $(\mathrm{n}=54)$} & \multicolumn{2}{c}{ Daycare assistants $(\mathrm{n}=56)$} & \multicolumn{2}{c}{ Total } & \multicolumn{2}{c}{$\mathrm{F}$} \\
& Mean & SD & Mean & SD & Mean & SD & Mean & SD & . \\
\hline MI & 29.485 & 6.99 & 29.740 & 6.37 & 31.01 & 6.57 & 30.04 & 6.67 & $.889^{*}$ \\
AV & 114.74 & 9.412 & 110.69 & 12.428 & 108.75 & 13.039 & 111.62 & 11.799 & $4.356^{* *}$ \\
AN & 85.74 & 6.836 & 85.98 & 6.503 & 84.57 & 5.969 & 85.44 & 6.464 & $.764^{*}$ \\
PV & 60.40 & 4.601 & 60.67 & 4.682 & 59.52 & 4.969 & 60.20 & 4.741 & $.899^{*}$ \\
\hline
\end{tabular}

\subsection{Childcare predictions of moral intelligence}

The prediction of childcare towards moral intelligence was tested using SPSS 20.0 software with simple regression analysis techniques. All types of childcare predicted moral intelligence $(\mathrm{R}=.304, \mathrm{R} 2=.092$, $\mathrm{F}=5.908, \mathrm{p}<.01)$. Specifically, the type of authoritative childcare has a significant effect on moral intelligence of young children $(\beta=.258, \mathrm{t}=3.407, \mathrm{p}<.01)$. Permissive childcare types also have a significant effect on moral intelligence $(\beta=.162, t=2.000, p<.05)$. Meanwhile, the type of authoritarian childcare has no effect on moral intelligence $(\beta=-.079, \mathrm{t}=-0.945, \mathrm{p}>.05)$. Predictions of childcare towards moral intelligence are presented in Table 2.

Table 2. Childcare model prediction on moral intelligence

\begin{tabular}{lccccc}
\hline The direction of the relationship & $\mathrm{R}$ & $\mathrm{R}^{2}$ & $\mathrm{~F}$ & $\beta$ & $\mathrm{t}$ \\
\hline Model & .304 & .092 & $5.908^{* * *}$ & & \\
AV $\rightarrow$ MI & & & & .258 & $3.407^{* * *}$ \\
AN $\rightarrow$ MI & & & & -.079 & $-0.945^{*}$ \\
PV $\rightarrow$ MI & & & .162 & $2.000^{* *}$ \\
\hline
\end{tabular}




\subsection{Moderator effects of non-parental childcare on childcare patterns and moral intelligence}

The regression test results show that authoritative and permissive childcare have a significant effect on the moral intelligence of young children. Furthermore, a moderation analysis was carried out to determine the role of the moderator variable in the relationship between authoritative and permissive childcare in moral intelligence. The moderating variables were the grandmother's childcare group, household assistants' childcare, and daycare assistants' childcare. The analysis was tested using PROCESS Macro on SPSS 20.0 software. A summary of the results of the moderation analysis is presented in Table 3.

Table 3. Bootstrapped conditional effects of childcare on moral intelligence in terms of care between 'grandmothers' childcare, household assistants' childcare, and daycare assistants' childcare

\begin{tabular}{cccccc}
\hline $\begin{array}{c}\text { Effect of moderating } \\
\text { variables }\end{array}$ & Estimate & SE & Lower & $\begin{array}{c}\text { Observed moderated effects } \\
\text { Upper }\end{array}$ & $\mathrm{p}$ \\
\hline AV $\rightarrow$ MI & .00 & .05 & -.09 & .10 & .88 \\
PV $\rightarrow$ MI & -.31 & .12 & -.55 & -.06 & .01 \\
\hline The effect of permissive parenting on moral intelligence on care between grandmothers, household \\
arsistants, and daycare assistants \\
Grandmothers & .57 & .16 & .25 & .88 & .00 \\
Household assistants & .26 & .10 & .05 & .46 & .01 \\
Daycare assistants & .05 & .16 & -.37 & .26 & .74 \\
\hline
\end{tabular}

The results of the moderation analysis show that there is a moderating effect on the relationship between permissive childcare and moral intelligence $(\beta=-.31, p<.05)$. There is no moderating effect in the relationship between authoritative childcare and moral intelligence $(\beta=.00, \mathrm{p}>.05)$.

The results of the analysis of interaction effect in Table 3 showed that the influence of permissive childcare on moral intelligence is reported in the grandmothers' childcare group and the household assistants' childcare group. Meanwhile, in the daycare assistants' childcare group, there was no influence of permissive childcare on moral intelligence. The moderator effect of non-parental childcare upbringing on permissive childcare and moral intelligence can be seen in Figure 1 of the interaction patterns (scatterplot).

Figure 1 shows that in the grandmother's childcare, there was an interaction between permissive childcare towards early childhood moral intelligence. Likewise, in the household assistants' childcare, there was also a permissive childcare interaction with early childhood moral intelligence. This shows that the higher the permissive childcare applied by grandmothers' childcare and household assistants' childcare, the higher the moral intelligence of the young children. However, in the daycare assistants' childcare, there was no interaction between permissive childcare and moral intelligence.

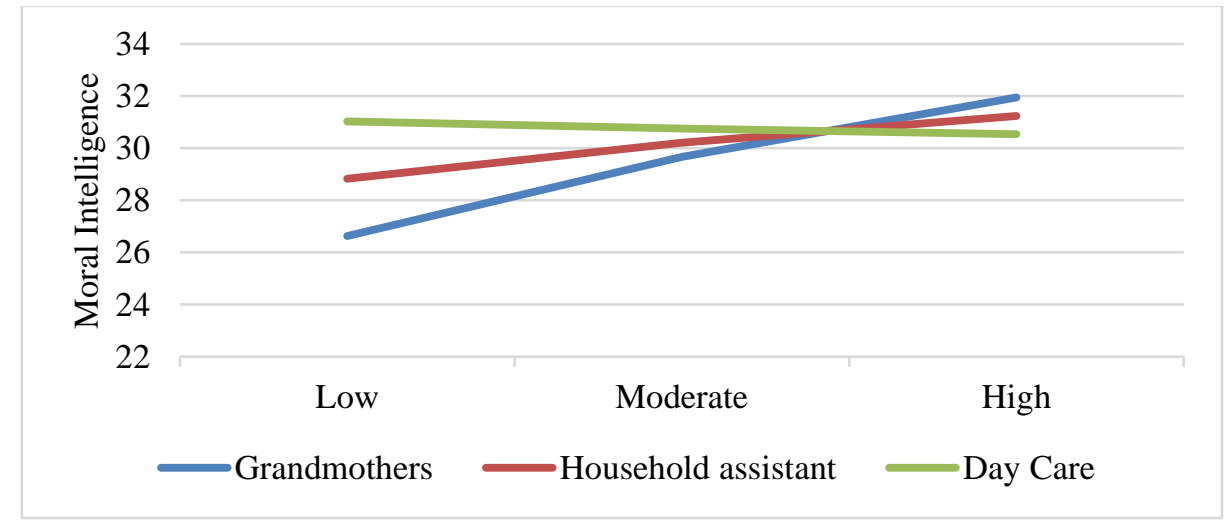

Figure 1. Interaction pattern graph

\section{DISCUSSION}

This study examined non-parental childcare on the development of moral intelligence in early childhood, considering that currently many children are under non-parental childcare. The effects of parenting on moral intelligence have been analyzed by previous studies [14]-[16]. However, the study was conducted on parents and only measured one particular type of childcare. Meanwhile, this study analyzes the effect of non- 
parental childcare on the moral intelligence of early childhood. This study explores three types of childcare (authoritarian, authoritative, and permissive childcare) [27] implemented by the non-parental childcare to the development of moral intelligence. The results indicate that authoritative and permissive childcare practices by non-parental childcare have a significant effect on the moral intelligence of early childhood. Furthermore, it also indicates that authoritative childcare has a stronger effect on moral intelligence in early childhood when compared to permissive childcare.

Previous research showed that permissive childcare does not affect children's moral development [28]. It also showed that permissive childcare has a negative impact on children's behavior. According to the research by Masud, et al. [29], permissive childcare produces aggressive and negative behavior in children. Hosokawa and Katsura [30] research shows that permissive childcare influences behavior problems in boys. Meanwhile, research by Tan and Yasin [31], Shahsavari [32] shows that permissive childcare are not effective in encouraging children's morals.

Interestingly, this study contributed to clarifying that permissive childcare does not always have a negative impact on the development of moral intelligence. Permissive childcare also has a positive effect on the culture in which the childcare is applied, for a collectivistic culture such as in Indonesia, the effect of permissive childcare can be positive. Other research has shown that the negative influence of permissive childcare on moral has a point, but not always because it depends on the cultural context of the local community [33]. The effect of permissive childcare on children's moral intelligence in this study was greater in the grandmothers' childcare than in the household assistants' childcare.

The results showed that permissive childcare has an advantage in the development of children's moral intelligence in grandmothers' childcare and household assistants' childcare. As it is known, the care of grandmothers and household assistants tends to be personal. Furthermore, personal childcare encourages children to explore moral knowledge in which children can develop reflections on the use of moral values. The conditions that arise in permissive childcare are that adults communicate the consequences of behavior and discusses the rules to the children, and not just providing moral rules [34], [35]. Stating that in permissive childcare, adults consider children as partners so that fewer restrictions are imposed. Meanwhile, in mass parenting conditions, the results showed that children can learn moral through enforcing rules. Compliance with the rules for mass childcare in accordance with Kohlberg's theory of moral development [36], [37] at the pre-conventional stage, according to which the children's moral intelligence is related to social norms that result from the children's relationships with their environment. This moral enforcement only occurs when children are with other people [38].

\section{CONCLUSION}

The results showed that parenting childcare affects moral intelligence. Authoritative childcare has a more significant impact on moral intelligence when compared to permissive childcare. Furthermore, it reported that grandmothers' childcare and household assistants' childcare is more likely to lead to permissive childcare, which is more flexible and enforces the freedom to apply rules to children because they only care for a few children. Meanwhile, daycare assistants' childcare needs to use firmness in applying rules to children because they care for more children.

This study has several limitations. First, related to the sample, which includes young children aged 56 , and a total of 178 children from 11 schools in different districts. Therefore, a larger sample covering all regions is needed in order to provide more representative results. This study only examined the effect of nonparental childcare upbringing on the development of early childhood moral intelligence, further studies can focus on the effect of non-parental childcare upbringing on the development of another intelligence.

The practical implication of this study relates to the development of moral intelligence in early childhood by non-parental childcare. In this regard, the care of grandmothers' childcare and household assistants' childcare needs to improve permissive childcare because they are more flexible and provide freedom in applying rules and considering children's opinions in developing moral intelligence. Meanwhile, daycare assistants' childcare needs to be firm in applying the rules because they care for more children. This study suggests that further research should examine the effects of non-parental childcare on other intelligence and examine aspects of moral intelligence more closely using a larger sample.

\section{ACKNOWLEDGEMENTS}

This research is funded by Direktorat Riset dan Pengabdian Masyarakat Kemenristekdikti funding in 2020. 


\section{REFERENCES}

[1] J. G. Smetana, "Becoming moral: Introduction to the special issue on early moral development," Hum. Dev., vol. 61, no. 4-5, pp. 209-213, 2018.

[2] N. Eskandari et al., "Explaining the Relationship between Moral Intelligence and Professional Self-Concept with the Competency of Nursing students in Providing Spiritual Care to Promote Nursing Education," J. Educ. Health Promot., vol. 8, pp. 1-6, 2019.

[3] J. M. A. M. Janssens and D. Maja, "Parents' Child-Rearing Style and Child's Sociometric Status," Dev. Psychol., vol. 28 , no. 5, pp. 925-932, 1992.

[4] O. I. Olusola and A. O. Samson, "Moral Intelligence: An Antidote to Examination Malpractices in Nigerian Schools," Univers. J. Educ. Res., vol. 3, no. 1, pp. 32-38, 2015.

[5] M. Beheshtifar, Z. Esmaeli, and M. N. Moghadam, "Effect of Moral Intelligence on Leadership," Eur. J. Econ. Financ. Adm. Sci., vol. 43, no. 43, pp. 6-11, 2011.

[6] M. Raisi, H. A. Tehran, S. Bakouei, and S. Momenuan, "Moral Intelligence and Aggression in Students," J. Biostat. Epidemiol., vol. 4, no. 1, pp. 1-9, 2018.

[7] M. Toprak and M. Karakus, "Teachers' moral intelligence: A scale adaptation into Turkish and preliminary evidence," Eur. J. Educ. Res., vol. 7, no. 4, pp. 901-911, 2018.

[8] M. Borba, Building moral intelligence: The seven essential virtues that teach kids to do the right thing. Jossey-Bass, 2002.

[9] J. W. Santrock, Child development, (in Bahasa). Erlangga: Jakarta, 2007.

[10] H. M. Beißert and M. Hasselhorn, "Individual differences in moral development: Does intelligence really affect children's moral reasoning and moral emotions?" Front. Psychol., vol. 7, pp. 1-10, Dec. 2016.

[11] L. Fransisca and C. R. P. Ajisuksmo, "The correlations among moral knowing, moral feeling, and moral behavior on four basic competencies of teachers," (in Bahasa), J. Kependidikan Penelit. Inov. Pembelajaran, vol. 45, no. 2, pp. 211-221, 2015.

[12] M. Khosravani, M. Khosravani, F. Borhani, and M. Mohsenpour, "The relationship between moral intelligence and organizational commitment of nurses," Clin. Ethics, vol. 15, no. 3, pp. 126-131, 2020.

[13] M. Christen and D. Narvaez, "Moral Development in Early Childhood is Key for Moral Enhancement," AJOB Neurosci., vol. 3, no. 4, pp. 25-26, 2012.

[14] K. Campaert, N. Annalaura, and M. Ersilia, "The Role of Poor Parenting and Parental Approval for Children's Moral Disengagement," J. Child Fam. Stud., vol. 27, pp. 2656-2667, 2018.

[15] M. E. Augustine and C. A. Stifter, "Temperament, parenting, and moral development: Specificity of behavior and context," Soc. Dev., vol. 24, no. 2, pp. 285-303, 2015.

[16] R. Karmakar, "Does Parenting Style Influence the Internalization of Moral Values in Children and Adolescents ?" Psychol Stud, vol. 60, pp. 438-446, Dec. 2015.

[17] A. Alhadabi, S. Aldhafri, H. Alkharusi, I. Al-Harthy, M. Alrajhi, and H. AlBarashdi, "Modelling parenting styles, moral intelligence, academic self-efficacy and learning motivation among adolescents in grades 7-11," Asia Pacific J. Educ., vol. 39, no. 1, pp. 133-153, 2019.

[18] International Labour Organization, ILOSTAT, "Labor force participation rate, female (\% of female population ages 15+) (modeled ILO estimate) Data," The World Bank, 2020. [Online]. Available: https://data.worldbank.org/indicator/SL.TLF.CACT.FE.ZS.

[19] E. W. Latifah, K. Diah, and P. Herien, "The Influence of Mothers' and Grandmothers' Parenting on the Preschoolage Childrens' Independence and Cognitive Development," (in Bahasa), Jur. Ilm. Kel. Kons, vol. 9, no. 1, pp. 21-32, 2016, doi: 10.24156/jikk.2016.9.1.21.

[20] S. S. Supsiloani, Puspitawati, and N. Hasanah, "The Existence of Children's Daycare and its Benefits to the Working Housewives (A Case Study at Dharma Asih Children's Daycare in Medan City)," (in Bahasa), Jupiis J. Pendidik. Ilmu-Ilmu Sos, vol. 7, no. 2, pp. 119-124, 2015, doi: 10.24114/jupiis.v7i2.3117.

[21] S. Ma, E. E. Chen, and H. Li, "Foreign Domestic Helpers' Involvement in Non-Parental Childcare: A Multiple Case Study in Hong Kong," J. Res. Child. Educ., vol. 34, no. 3, pp. 427-446, 2020.

[22] C. Zhang, et al., "How urban Chinese parents with 14-month-old children talk about nanny care and childrearing ideals," J. Fam. Stud., vol. 26, no. 4, pp. 611-627, 2020.

[23] M. L. S. De Moura, L. F. Pessôa, D. De Oliveira Ramos, D. M. L. F. Mendes, A. C. M. Fioravanti-Bastos, and L. B. T. Dias, "Beliefs of mothers, nannies, grandmothers and daycare providers concerning childcare," Paideia, vol. 24, no. 59, pp. 341-349, 2014.

[24] I. Loudová and J. Lašek, "Parenting Style and its Influence on the Personal and Moral Development of the Child," Procedia - Soc. Behav. Sci., vol. 174, pp. 1247-1254, 2015.

[25] S. M. Shadmanfaat, J. Choi, S. Kabiri, and I. Yun, "Assessing the Links Between Parenting Practices, Moral Emotions, and Cyberbullying Perpetrations among a Sample of Iranian Sports Fans," Int. J. Offender Ther. Comp. Criminol., vol. 64, no. 16, pp. 1674-1695, 2020.

[26] C. C. Robinson, M. Barbara, F. O. Susanne, and H. H. Craig, "Authoritative, Authoritarian, and Permissive Parenting Practices: Development of A New Measure,” Psychol. Rep., vol. 77, pp. 819-830, 1995.

[27] D. Baumrind, "Effects of Authoritative Parental Control on Child Behavior," Child Development, vol. 37, no. 4, pp. 887-907 1966.

[28] Z. Babajanzadeh and A. K. Mohammadi Otaghsara, "Investigate the Relationship between Parenting Styles with Development of Moral Judgment in Children 4-6 Years in Kindergartens of the City of Babol," Mediterr. J. Soc. Sci., vol. 7, no. 2, pp. 92-99, 2016. 
[29] H. Masud, M. S. Ahmad, K. W. Cho, and Z. Fakhr, "Parenting Styles and Aggression Among Young Adolescents: A Systematic Review of Literature," Community Ment. Health J., vol. 55, no. 6, pp. 1015-1030, 2019.

[30] R. Hosokawa and T. Katsura, "Role of parenting style in children's behavioral problems through the transition from preschool to elementary school according to gender in Japan," Int. J. Environ. Res. Public Health, vol. 16, no. 1, 2019.

[31] W. N. Tan and M. Yasin, "Parents' roles and parenting styles on shaping children's morality," Univers. J. Educ. Res., vol. 8, no. 3, pp. 70-76, 2020.

[32] M. Shahsavari, "A general overview on parenting styles and its effective factors," Aust. J. Basic Appl. Sci., vol. 6, no. 8, pp. 139-142, 2012.

[33] R. Forehand and B. A. Kotchick, "Cultural diversity: A wake-up call for parent training," Behav. Ther., vol. 27, no. 2, pp. 187-206, 1996.

[34] R. L. Selman, "The relation of role taking to the development of moral judgment in children," Child Dev., vol. 42, no. 1, pp. 79-91, 1971.

[35] H. Kotaman, "Freedom and Child Rearing: Critic of Parenting Practices from a New Perspective," Procedia - Soc. Behav. Sci., vol. 82, pp. 39-50, 2013.

[36] N. Porter, "Kohlberg and Moral Development," J. Moral Educ., vol. 1, no. 2, pp. 37-41, 1972.

[37] Q. Zhang and H. Zhao, "An Analytical Overview of Kohlberg's Theory of Moral Development in College Moral Education in Mainland China," Open J. Soc. Sci., vol. 05, no. 08, pp. 151-160, 2017.

[38] J. A. Merin, "Practical Review and Evaluation on Parenting Education," Int. J. Eval. Res. Educ. (IJERE), vol. 7, no. 3, pp. 253-258, 2018. 